
allemande

$52-2 \mid 2020$

Les traités de paix en Europe centrale : quels potentiels pour quelles réalisations?

\title{
Fatima SAADI, Diderot et Lessing. La configuration de la
} scène moderne

Paris, L'Harmattan, 2020

Jean-Jacques Alcandre

\section{(2) OpenEdition}

Édition électronique

URL : https://journals.openedition.org/allemagne/2480

DOI : 10.4000 /allemagne. 2480

ISSN : 2605-7913

Éditeur

Société d'études allemandes

Édition imprimée

Date de publication : 31 décembre 2020

Pagination : 443-444

ISSN : 0035-0974

Référence électronique

Jean-Jacques Alcandre, "Fatima sAADI, Diderot et Lessing. La configuration de la scène moderne ", Revue d'Allemagne et des pays de langue allemande [En ligne], 52-2 | 2020, mis en ligne le 31 décembre 2020, consulté le 20 février 2022. URL : http://journals.openedition.org/allemagne/2480 ; DOI : https:// doi.org/10.4000/allemagne.2480 


\section{Italiques}

Fatima SAADI, Diderot et Lessing. La configuration de la scène moderne, Paris, L'Harmattan (coll. Univers théâtral), avril 2020, 253 p.

L'ouvrage de Fatima Saadi est une réécriture de sa thèse de doctorat soutenue il y a plus de vingt ans (La configuration de la scène moderne, 1998). II bénéficie par là même de ces nombreuses années qui ont encore enrichi sa profonde connaissance de l'histoire et de la théorie dramatique, mais aussi de sa pratique de la création théâtrale, puisqu'elle exerce la fonction de Dramaturg à la compagnie brésilienne Teatro do Pequeno Gesto depuis sa création en 1991. Cette double compétence à un tel point d'excellence est bien rare et mérite donc d'être soulignée dès le début de cette introduction.

Car l'enjeu de cette étude est précisément de se situer à l'articulation entre les écrits théoriques, les textes théâtraux et la concrétisation scénique du spectacle théâtral. Or cette mise en relation n'a rien d'une évidence. Elle est le résultat d'une évolution, d'une conquête de l'autonomie de la création théatrale par rapport au champ de la littérature: la phase de réalisation du spectacle théâtral prend toute son importance dans le théâtre européen à partir du milieu du XVIIle siècle, ceci tout particulièrement à travers les ceuvres dramatiques et les travaux théoriques de Denis Diderot et de Gotthold Ephraim Lessing. Fatima Saadi nous livre ainsi l'histoire d'une émancipation: le théâtre du XVIIIe siècle se libère de la tutelle du classicisme français lui-même fondé sur une interprétation particulière de la théorie et de l'écriture dramatique de l'Antiquité classique.

La confrontation théoricienne de Diderot et Lessing avec Aristote est donc essentielle, mais aussi l'importance décisive attribuée à la réalisation scénique et au jeu de l'acteur (notamment le renouveau de Shakespeare à travers les interprétations de David Garrick). Mais ce mouvement d'émancipation passe aussi par une approche globale des faits artistiques qui abat les cloisons entre les disciplines dans le domaine de l'art. Diderot, à travers ses références et analyses dans le domaine de la peinture, Lessing, avec sa prise d'appui sur les arts plastiques (statutaire), enclenchent un mouvement qui fera plus tard de la représentation théatrale un spectacle global dont l'écriture textuelle ne représente qu'un des éléments constitutifs et qui permet à l'œuvre écrite de 
"renaître» à chaque série de représentations. Ce sera alors le passage officiellement assumé à l'ère de la mise en scène (fin du XIXe siècle) que Fatima Saadi présente et analyse dans son indispensable conclusion.

Lire cet ouvrage permet donc d'avoir un regard privilégié sur une phase décisive de l'évolution du théâtre européen, mais aussi sur la création théâtrale en général à travers la multiplicité de ses constituants textuels et scéniques. Enfin, et ce n'est pas le moins important, il nous permet de constater à quel point la création théâtrale et la réflexion qui l'accompagne sont un marqueur privilégié, voire même un acteur direct de l'évolution sociale et de l'échange interculturel. Le XVIIle siècle européen est à cet égard d'une richesse étonnante et il mérite de ce fait toute notre attention, en un temps où la peur de l'autre et le repli sur soi semblent de plus en plus motiver les esprits à l'échelle du monde. Toute notre reconnaissance donc à Fatima Saadi d'avoir porté ce regard à la fois aigu et passionné sur la création théâtrale européenne.

Jean-Jacques Alcandre

Peter-Paul BÄNZIGER, Die Moderne als Erlebnis. Eine Geschichte der Konsum- und Arbeitsgesellschaft 1840-1940, Göttingen, Wallstein Verlag, 2020, 452 p.

Peter-Paul Bänziger, qui enseigne actuellement en histoire contemporaine à l'Université de Bâle, nous livre dans cet ouvrage une histoire du vécu quotidien. Son enquête porte sur l'espace germanophone pendant la période de modernisation qui s'étend des révolutions de 1848 à la Seconde Guerre mondiale. Il a étudié pour cela une centaine de journaux intimes et de recueils de lettres conservés dans divers centres d'archives comme le Leo Baeck Institute de New York, le Deutsches Tagebuch Archiv d'Emmendingen, ou la collection Frauennachlässe de Vienne. Ces ego-documents, qui émanent surtout d'un milieu d'artisans et de bourgeois, retracent des destinées multiples. L'auteur les a fondues dans une sorte de biographie collective structurée selon un plan thématique, de façon à faire ressortir l'évolution d'une culture subjective commune. À travers des conditions de vie différentes, les contemporains partagent un même répertoire de représentations et de modes d'action.

L'exposé, très détaillé, transporte le lecteur dans un monde parfois éloigné du nôtre, tant pour les modes de vie que pour les façons de penser, mais sur une période suffisamment longue pour nous faire saisir toute l'évolution qui a abouti à notre présent. Sans chercher à appliquer les techniques statistiques à son corpus de données, l'auteur recourt à la méthode du commentaire littéraire. Son étude, solidement structurée et confrontée à une riche bibliographie, constitue une preuve, s'il en était besoin, de la valeur heuristique que possède une explication de texte riche de comparaisons et de nuances. Peter-Paul Bänziger nous fait pénétrer au plus intime de la vie personnelle de ses personnages, artisans, enfants d'industriels, pasteurs, employés, domestiques, etc. Les transformations de leur vécu sont décrites en considérant deux domaines essentiels, celui de la production et celui de la consommation.

La modernisation s'accélère durant les deux décennies de la fin du XIXe siècle. Les journaux personnels s'inscrivent moins que par le passé dans la perspective à long terme d'une biographie et davantage dans le vécu instantané (Erlebnis). Les éléments de permanence s'estompent. La maison familiale perd sa fonction ancienne de lieu de production et de consommation. Le logement se déconnecte du lieu de travail. L'ethos 